\title{
Nitrofurantoin-associated bronchiolitis obliterans organizing pneumonia: Report of a case
}

\author{
Mark E Fenton MSc MD FRCPC ${ }^{1}$, Rani Kanthan MBBS FRCPC ${ }^{2}$, Donald W Cockcroft MD FRCPC
}

\begin{abstract}
ME Fenton, R Kanthan, DW Cockcroft. Nitrofurantoinassociated bronchiolitis obliterans organizing pneumonia: Report of a case. Can Respir J 2008;15(6):311-312.

Bronchiolitis obliterans organizing pneumonia due to nitrofurantoin has rarely been reported and is associated with poor outcomes. A case of nitrofurantoin-associated bronchiolitis obliterans organizing pneumonia responsive to drug withdrawal and corticosteroids is presented.
\end{abstract}

Key Words: Bronchiolitis obliterans organizing pneumonia; Nitrofurantoin pulmonary toxicity

Titrofurantoin is a commonly prescribed antimicrobial that 1 has several documented side effects, including pulmonary toxicity (1-4). Pulmonary toxicity is mainly acute by nature (3), but chronic disease does occur. Historically, the most common pathological association with nitrofurantoin is interstitial pneumonitis and fibrosis, although a variety of pathologies have been reported, including nonspecific interstitial pneumonia and desquamative interstitial pneumonia (1). Bronchiolitis obliterans organizing pneumonia (BOOP) is uncommon and has rarely been associated with nitrofurantoin use. There are six reported cases of nitrofurantoin-associated BOOP (1,5-7). We present an additional case of nitrofurantoin-associated BOOP.

\section{CASE PRESENTATION}

A 71-year-old female nonsmoker presented with one year of progressively increasing dry cough, fever, weight loss and exertional dyspnea. She was admitted to hospital with nonresolving pneumonia. Her past medical history was remarkable for remote membranoproliferative glomerulonephritis, hypothyroidism and osteopenia. She had normal renal function and had been taking nitrofurantoin for recurrent urinary tract infections for six months preceding the onset of symptoms.

Her respiratory rate was 20 breaths/min and room air oxygen saturation was 93\%. Auscultation revealed decreased air entry predominantly in the left lung with bibasilar inspiratory crackles. The remainder of the examination was unremarkable except for mild lower-extremity edema.

A complete blood count was remarkable for mildly elevated monocytes. Antinuclear antibody was positive at a titre greater than 1:1280 with speckled and nucleolar pattern. Antineutrophil cytoplasmic antibody screen and rheumatoid factor were normal, and erythrocyte sedimentation rate was $56 \mathrm{~mm} / \mathrm{h}$. Serum creatinine was $80 \mu \mathrm{mol} / \mathrm{L}$. Computed tomography of the chest demonstrated marked bronchial wall

\section{Une bronchiolite oblitérante avec pneumonie organisée associée à la nitrofurantoïne : Rapport de cas}

\begin{abstract}
La bronchiolite oblitérante avec pneumonie organisée causée par la nitrofurantoïne est rarement déclarée et est reliée à une mauvaise issue. Les auteurs présentent un cas de bronchiolite oblitérante avec pneumonie organisée associée à la nitrofurantoïne sensible au sevrage des médicaments et aux corticoïdes.
\end{abstract}

thickening, fissural thickening, peripheral fibrosis in the mid and upper lung zones and pleural effusions. Overall, symptoms were believed to be consistent with BOOP. Thoracentesis demonstrated unremarkable transudative fluid. Video-assisted thoracoscopic biopsy was performed, and biopsy samples demonstrated proliferating fibromyomatous connective tissue with early organization within the air spaces, consistent with BOOP (Figures 1 and 2). Pulmonary function tests demonstrated a forced expiratory volume in $1 \mathrm{~s}$ of $1.33 \mathrm{~L}$ ( $57 \%$ of predicted) and a forced vital capacity of $1.53 \mathrm{~L}(47 \%$ of predicted), to give a ratio of 0.87 . Diffusing capacity was not measured initially because the patient was too ill to perform the manoeuvre.

Nitrofurantoin-induced lung disease was suspected and the drug was discontinued, concurrent with the initiation of prednisone $40 \mathrm{mg}$ daily. In the following months, there was gradual and essentially complete resolution of symptoms, as well as normalization of flows and volumes (Figure 3). However, a persistent moderate diffusing defect and minor radiographic changes remained. The first measured diffusing capacity, performed eight months after initiation of prednisone, was $49 \%$ of predicted and improved to $53 \%$ of predicted after the next 11 months. Prednisone was tapered over 16 months and discontinued.

\section{DISCUSSION}

This is the seventh reported case of nitrofurantoin-associated BOOP (1,5-7). Although similar in many ways to previously reported cases, it presents several important points. First, it underscores the treatable nature of nitrofurantoin-associated BOOP, with near complete resolution of disease on treatment. The residual fibrotic change and consequent diffusing defect may have been avoidable if there had been earlier recognition of the disease. However, a case reported by Cameron et al (6) suggested that the disease course is variable and that this

\footnotetext{
${ }^{1}$ Department of Medicine, Division of Respirology, Critical Care and Sleep Medicine; ${ }^{2}$ Department of Pathology, University of Saskatchewan, Saskatoon, Saskatchewan

Correspondence: Dr Donald W Cockcroft, Division of Respirology, Critical Care and Sleep Medicine, 103 Hospital Drive, Saskatoon,

Saskatchewan S7N OW8. Telephone 306-966-8275, fax 306-966-8694, e-mail don.cockcroft@usask.ca
} 


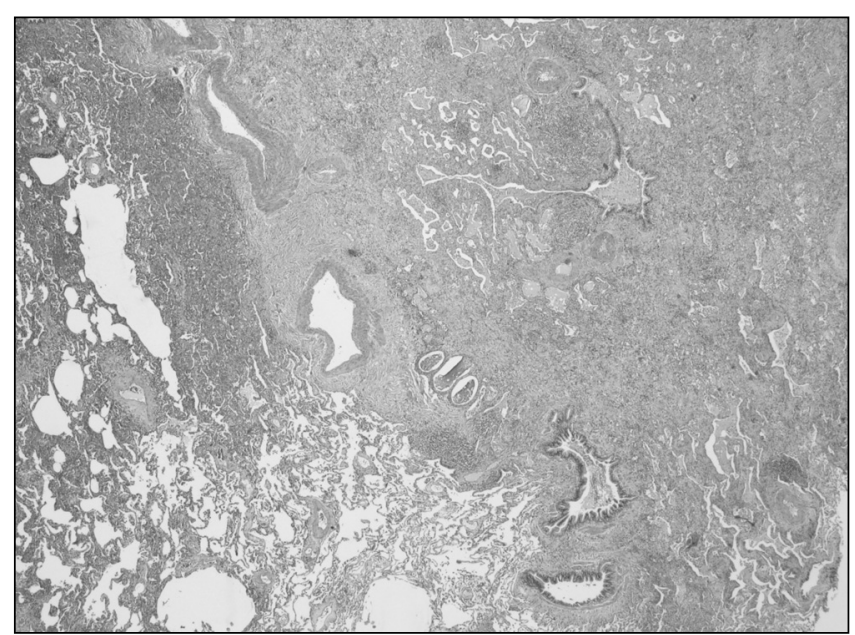

Figure 1) Low-power micrograph of a lung biopsy specimen. Open lung biopsy of the right upper lobe shows a spectrum of subacute and chronic changes. In some regions the lung shows loss of architecture with fibrosis, prominent chronic inflammation with an abrupt transition to architecturally normal lung without any honeycomb change. The bronchiolitis obliterans organizing pneumonia changes seen include areas with organizing pneumonia accompanied by intraluminal proliferation of polypoid fibromyxomatous connective tissue with scattered foci of chronic inflammatory cells throughout the interstitium. Mild thickening of the bronchiolar walls are seen together with moderately thick-walled vessels with no evidence of vasculitis. Original magnification $\times 40$

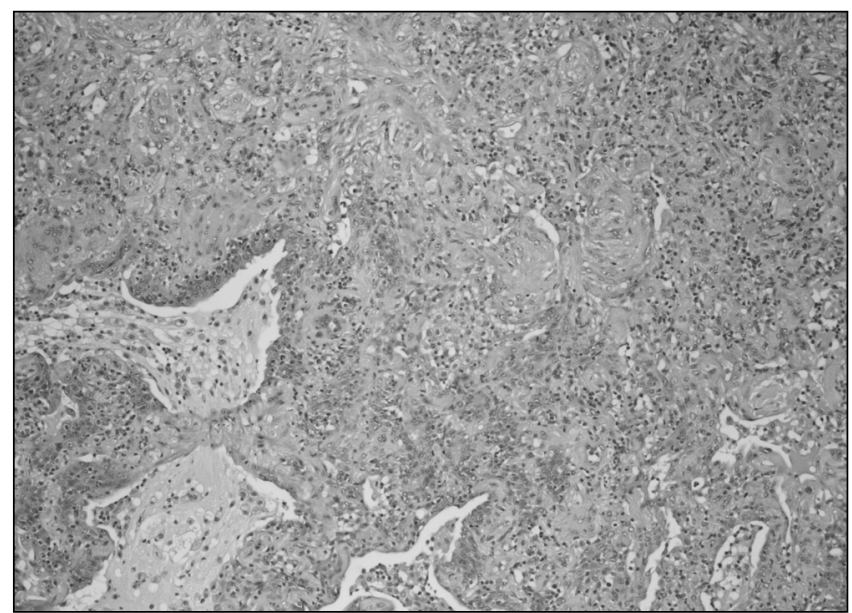

Figure 2) High-power micrograph of a lung biopsy specimen. The high-power microphotograph demonstrates the intraluminal proliferation of the fibromyxomatous connective tissue plugs and the diffuse chronic inflammation in the interstitium

hypothesis may not be true. Their patient had a shorter symptom duration, and had more pronounced spirometric and radiographic abnormalities that did not completely resolve with treatment. Another reported patient (5) did not respond well to treatment and died within eight to ten weeks of symptom onset. Both cases had a rapid prodrome of three to six weeks before diagnosis and both were ex-smokers. Therefore, two distinct forms of nitrofurantoin-associated BOOP - fulminant and subacute - may exist, with each having a unique clinical course and response to treatment. It is worth noting that one of the patients with fulminant and fatal respiratory failure had concurrent desquamative interstitial pneumonitis on biopsy, making it difficult to determine which entity was responsible for the demise of the patient (5). Our case had a subacute,

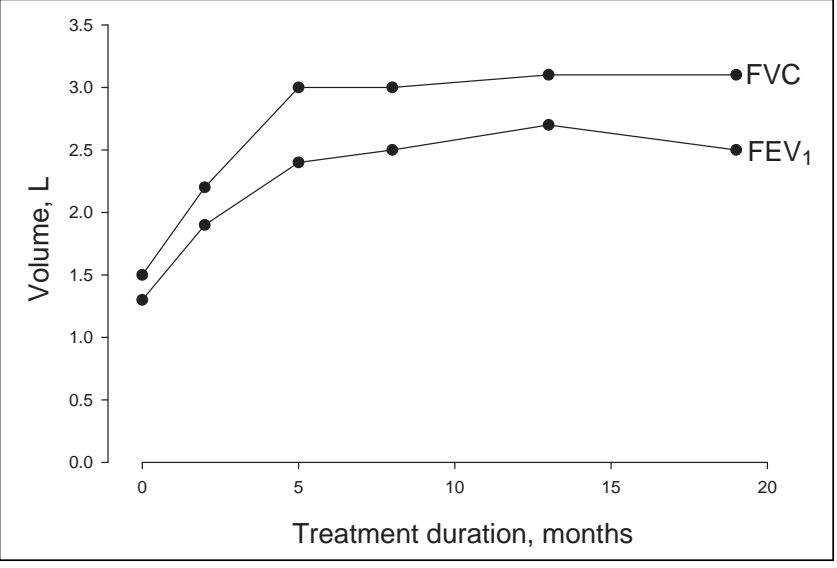

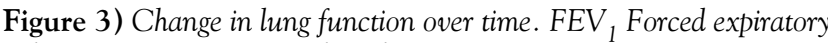
volume in $1 \mathrm{~s}$; FVC Forced vital capacity

progressive clinical course, BOOP on biopsy (Figures 1 and 2), and a marked clinical response to nitrofurantoin withdrawal and systemic corticosteroid treatment.

Cohen et al (5) postulated that an autoimmune diathesis may play a role in the genesis of BOOP in association with hypothyroidism, and this portends a poor prognosis. Our case, however, did not experience fulminant respiratory failure, as would be predicted by their hypothesis. It is possible that $\mathrm{BOOP}$ related to drug ingestion may differ pathogenetically from other forms of BOOP. We cannot rule out an association between $\mathrm{BOOP}$ and hypothyroidism, but if such an association exists in our patient, it did not result in a poor clinical outcome as previously suggested. We are unaware of any association between membranoproliferative glomerulonephritis and BOOP.

Despite alternative antibiotics for treatment of recurrent urinary tract infection, nitrofurantoin continues to be prescribed widely, probably due to its complete renal excretion. The present case, in conjunction with those previously reported, suggests there is a need for a high index of suspicion and vigilance in patients being treated with nitrofurantoin. In patients who experience respiratory symptoms, $\mathrm{BOOP}$ should be included in the differential diagnosis, particularly those on long-term treatment.

In summary, nitrofurantoin-associated $\mathrm{BOOP}$ is a rare entity that once recognized may respond well to drug withdrawal and treatment with steroids. Further studies are required to elucidate the pathophysiological mechanism of this disease.

\section{REFERENCES}

1. Mendez JL, Nadrous HF, Hartman TE, Ryu JH. Chronic nitrofurantoin-induced lung disease. Mayo Clin Proc 2005;80:1298-302.

2. Rosenow EC 3rd, DeRemee RA, Dines DE. Chronic nitrofurantoin pulmonary reaction. Report of 5 cases. N Engl J Med 1968;279:1258-62.

3. Holmberg L, Boman G, Böttiger LE, Eriksson B, Spross R, Wessling A. Adverse reactions to nitrofurantoin. Analysis of 921 reports. Am J Med 1980;69:733-8.

4. Pneumotox. <http://www.pneumotox.com> (Version current at June 25, 2008).

5. Cohen AJ, King TE Jr, Downey GP. Rapidly progressive bronchiolitis obliterans with organizing pneumonia. Am J Respir Crit Care Med 1994;149:1670-5.

6. Cameron RJ, Kolbe J, Wilsher ML, Lambie N. Bronchiolitis obliterans organising pneumonia associated with the use of nitrofurantoin. Thorax 2000;55:249-51.

7. Fawcett IW, Ibrahim NB. BOOP associated with nitrofurantoin. Thorax 2001;56:161. 


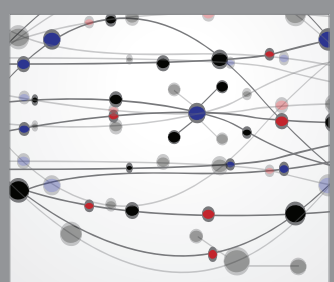

The Scientific World Journal
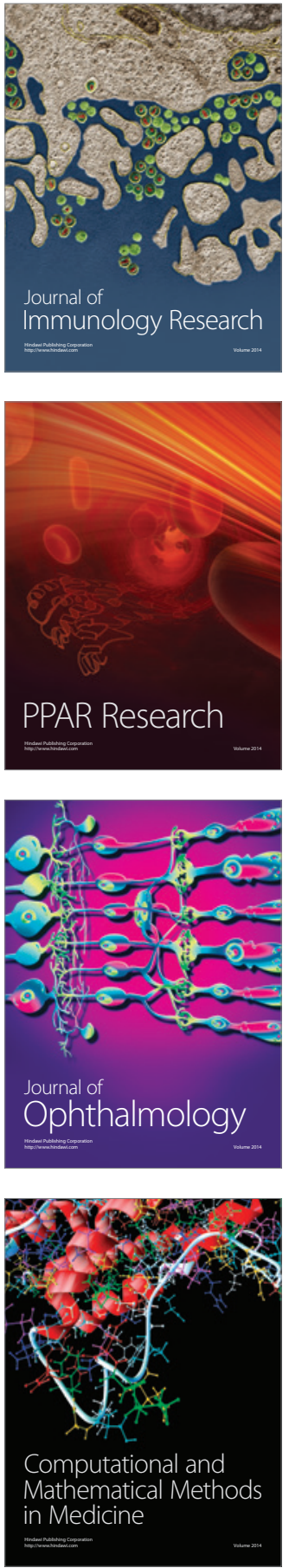

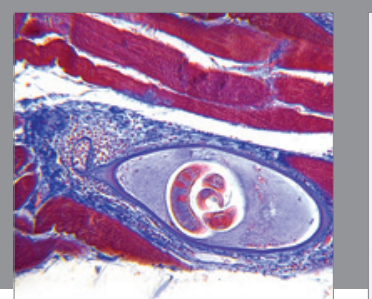

Gastroenterology Research and Practice

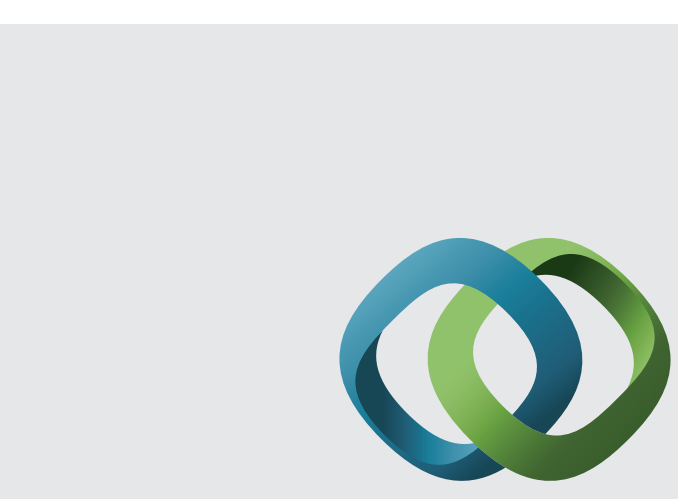

\section{Hindawi}

Submit your manuscripts at

http://www.hindawi.com
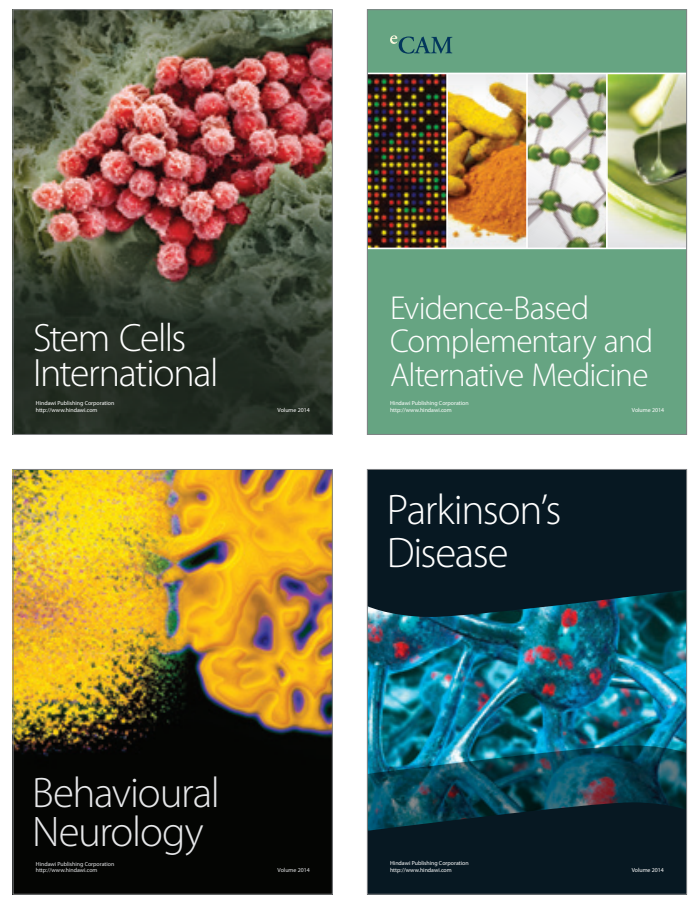
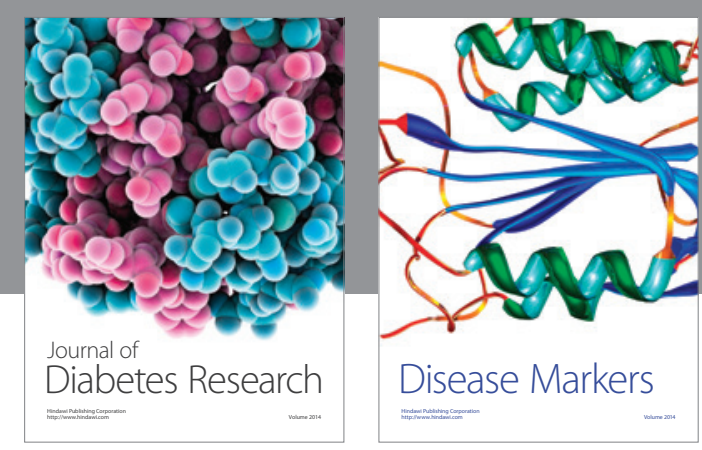

Disease Markers
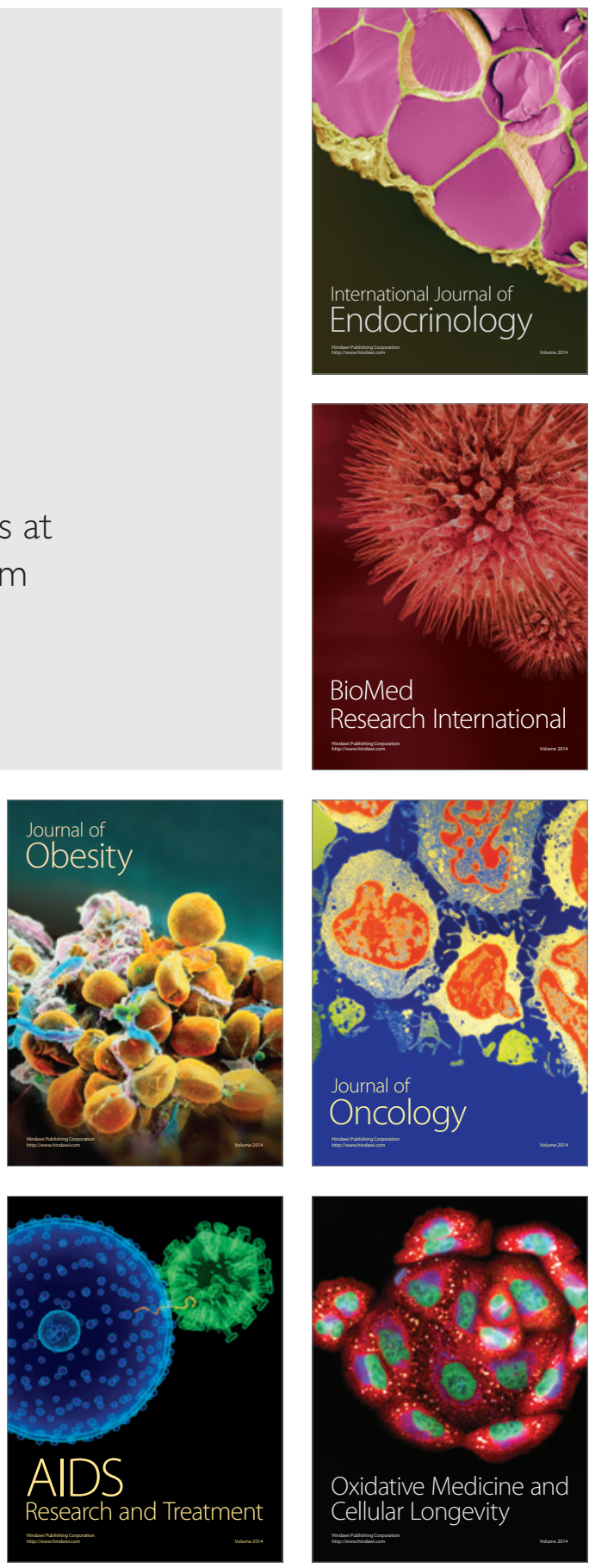